(C) 2020 IEEE. Personal use of this material is permitted. Permission from IEEE must be obtained for all other uses, in any current or future media, including reprinting/republishing this material for advertising or promotional purposes, creating new collective works, for resale or redistribution to servers or lists, or reuse of any copyrighted component of this work in other work.

\title{
Copper oxide coated D-shaped optical fibers for the development of LMR refractometers
}

\author{
Aritz Ozcariz $^{1}$, Ignacio Vitoria ${ }^{1}$, Francisco J. Arregui ${ }^{1,2}$, IEEE Member and Carlos R. Zamarreño ${ }^{1,2}$, Senior \\ Member, IEEE. \\ ${ }^{1}$ Dept. of Electrical, Electronic and Communications Engineering, Public University of Navarra, Pamplona 31006, Spain \\ ${ }^{2}$ Institute of Smart Cities, Jerónimo de Ayanz Building, UPNA, Pamplona, 31006, Spain \\ aritz.ozcariz@unavarra.es
}

\begin{abstract}
Lossy mode resonance (LMR) based refractometers obtained by means of copper oxide thin-films fabricated onto side-polished (D-shaped) are presented in this work. The high refractive index of copper oxide combined with the propagation mode isolation capabilities of $D$-shaped fibers allows for the observation of narrow $(30 \mathrm{~nm})$ and high sensitive $(10,336 \mathrm{~nm}$ per refractive index unit) LMRs, which could enable to improve the performance of LMR-based refractometers as well as provide an alternative label-free sensing platform for LMR-based sensors.
\end{abstract}

Keywords-optical fiber; LMR; lossy mode resonance; refractometer; optical sensor

\section{INTRODUCTION}

Lossy mode resonances (LMR) have been widely studied for the development of optical sensors [1-2]. The interaction between the light propagating through an optical fiber and a thin-film with a high refractive index and small absorption deposited on the fiber may lead to the generation of a LMR [3]. The central wavelength of such resonance will depend on the surrounding media refractive index (SRI). This way, an increase on the SRI will induce a red-shift on the LMR, making possible to stablish a direct relation between central LMR wavelength and SRI value. This technique has been used as the basis for the fabrication of a number of sensors [4-6].

LMRs are highly influenced by the optical properties of the thin-film [7]. This means that the nature of the coating material has an important impact on the performance of the sensor and the choice of the material is crucial for the optimization of LMR-based sensors. In this sense, a coating material with a high refractive index may increase the SRI sensitivity of the LMR devices, improving their performance [7]. For that purpose, this research studies the use of a high refractive index copper oxide thin-film for the development of LMR-based sensors. The device presented here is based on a D-shape fiber setup, which presents a side polished optical fiber segment where the coating is fabricated, which permits the interaction between the light propagating on the fiber and the deposited film. The use of polarized light in this fiber allows to observe the LMR components, transverse electric (TE) and transverse magnetic (TM), separately. This configuration helps to further optimize the performance of the sensors, as the isolation of modes permits to observe narrower resonances with higher sensitivities [8].

\section{MATERIALS AND METHODS}

The device consisted of a D-shaped fiber (Phoenix Photonics, Ltd., $1 \mathrm{~dB}$ of absorption at $1310 \mathrm{~nm}$ submerged in a medium of 1.5 refractive index) coated with a copper oxide thin-film by means of pulsed DC sputtering (Nadetech Inc.). This process is carried out at a partial Ar pressure of $2 \times 10^{-3}$ mbar and a current of $100 \mathrm{~mA}$, using a $\mathrm{CuO}$ target from Beijing Loyaltarget Technology Co.

During the coating fabrication, the transmission of the light at two different wavelengths will be monitored. For that purpose, the light source employed is a set of two lasers (BCP 400 A) connected to the D-shaped segment by a WDM for the fabrication probes (fig. 1a). An additional WDM is connected at the D-shaped segment output. The optical power is monitored using two optical power meters (RIFOCS $575 \mathrm{~L}$ ) connected at the WDM outputs and configured at the wavelength of each laser.

The refractometric characterization of the device has been performed using a broadband light source based on superluminescent emitting diodes (FJORD-X2-1330-1550 from Pyroistech S.A.) is connected to a linear polarizer and a manual polarization controller, before the connection to the D-shape fiber. The output light is received by an optical spectrum analyzer (OSA, HP 86142 A), as it can be seen on fig. 1b. In this setup a set of water/glycerol (Panreac Inc.) in varying proportions is used to study the spectral evolution of the resonances for different SRI. The refractive index of these solutions was measured 5 times and averaged using a Mettler Toledo Refracto 30GS commercial refractometer.

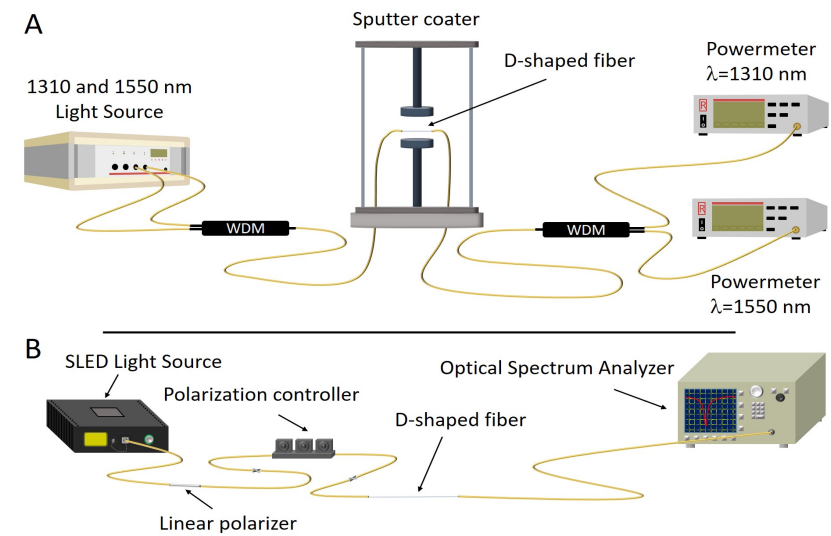

Figure 1: Setups used in the experiments to study (A) the transmission during the coating fabrication process and (B) the refractometric characterization. 


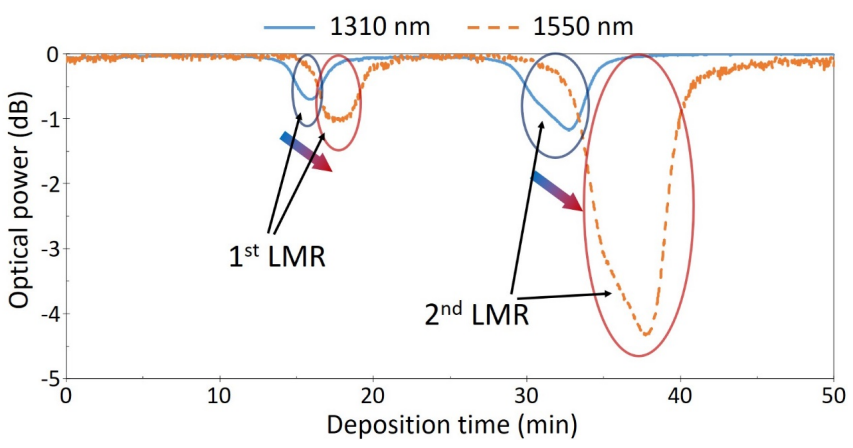

Figure 2: Evolution of the transmitted power at the wavelengths of 1310 $\mathrm{nm}$ and $1550 \mathrm{~nm}$ during the deposition of the copper oxide coating. The observed transmission dips are caused by the generation of LMRs as the film grows.

\section{RESULTS AND DISCUSSION}

The monitorization of the transmitted power at 1310 and $1550 \mathrm{~nm}$ allowed to observe the generation of LMRs as a function of the copper oxide thin-film thickness.

Fig. 2 represents the normalized optical power measured at 1310 and $1550 \mathrm{~nm}$ during a copper oxide deposition process of 50 minutes. Two LMRs can be separately observed. A first LMR is observed at $1310 \mathrm{~nm}$ after 15 minutes of deposition approximately and next it is observed at $1550 \mathrm{~nm}$. After 30 minutes, as the copper oxide film thickens, a second LMR is observed in the same way, in this case with a greater amplitude particularly at $1550 \mathrm{~nm}$. Here, it is interesting to note that, although the first LMR is observed as a simple small dip in the transmitted power, the shape observed for the second LMR indicates the presence of the two components corresponding to the TE and TM modes. This is seen in the fact that (for the second LMR), the power decreases fast at the beginning, then the slope changes abruptly and finally decreases at a slower rate until it reaches the minimum and it begins to rise again. The same behavior is seen both at 1310 and $1550 \mathrm{~nm}$ for the second LMR at a different moment during the deposition, which indicates that it was due to the overlap of two absorption peaks and not an effect of a change in the deposition rate. Another indicator of the presence of the two components in each LMR is the widening observed in the resonances when they are seen at $1550 \mathrm{~nm}$ compared to $1310 \mathrm{~nm}$, which is seen on both the first and the second LMR. One of the LMR components presents a greater shift and, as a result, the LMR widens as the thickness increases. As a consequence, each component presents slightly different sensitivity (to both SRI and film thickness).

An additional probe was obtained following previously described fabrication procedure. In this case, the fabrication process was stopped in order to observe a second order LMR when the D-shaped fiber is submerged on the water/glycerol solutions, achieved with a $105 \mathrm{~nm} \mathrm{CuO}$ thin-film. Such LMR was chosen due to the impossibility to tune a first order LMR in the desired wavelength and SRI ranges, caused by its extremely high sensitivity. Here, it is important to remark that the fabrication process is performed in air $(\mathrm{SRI}=1)$ while the tests are performed in aqueous solution $(\mathrm{SRI}=1.33)$ and small changes in thickness associated to the previously mentioned high sensitivity difficult the appropriate tuning of the LMR in the working wavelength range.

The spectra obtained when the second device is submerged in different RI solutions can be seen on Fig. 3. This graph shows the spectrum obtained in air $(\mathrm{SRI}=1)$, which presents a LMR at the top limit of the spectral range. When the probe is tested in water/glycerol solutions with higher SRI, such LMR shifts out of the working wavelength range. Then, a second LMR of higher order appears for SRI values above 1.379 .

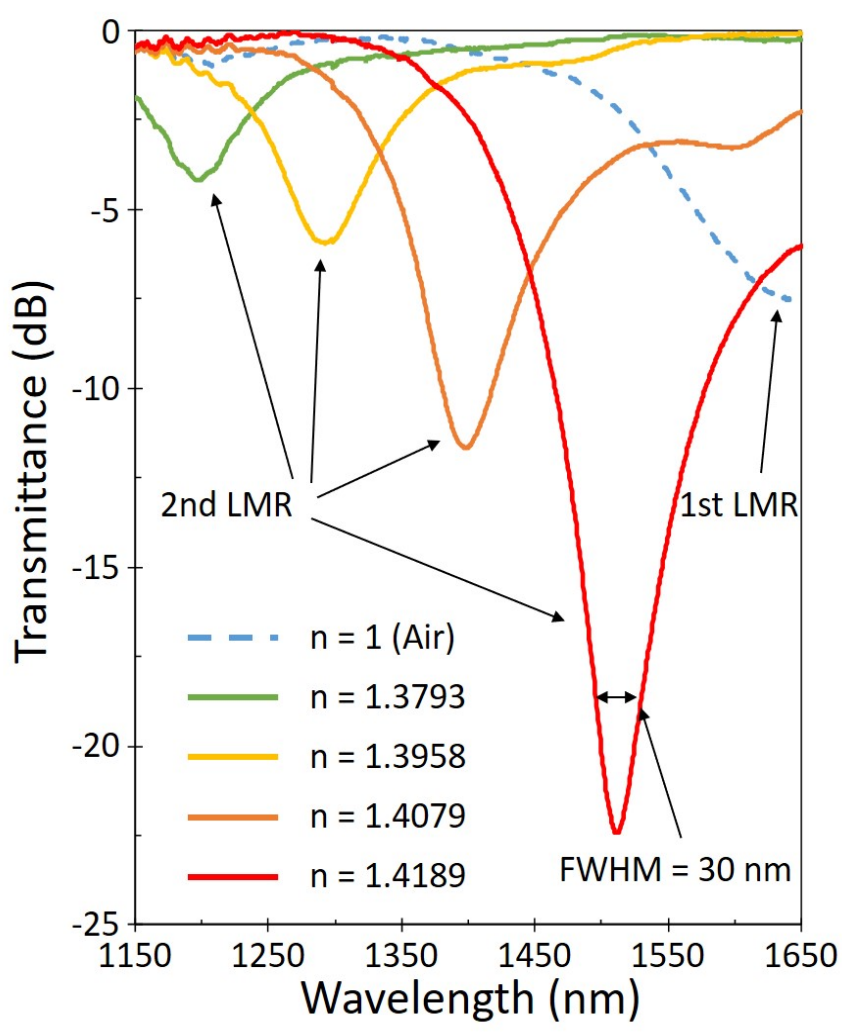

Figure 3: Transmittance spectra obtained with $105 \mathrm{~nm}$ thick $\mathrm{CuO}$ coated D-shaped fiber for various SRI values. 


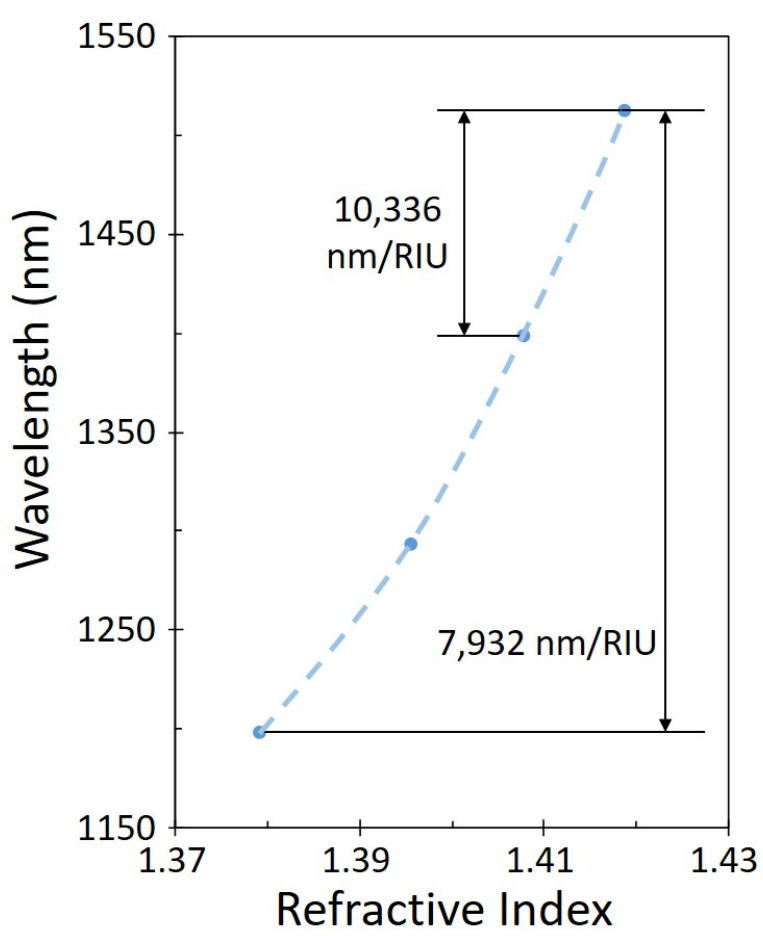

Figure 4: Calibration curve showing the central wavelength of the LMRs for the corresponding SRI value.

Due to the separation of the resonances, the LMR observed in air could be associated to one of the modes, presumably the TM mode, of a first order LMR, while the one observed for SRI values above 1.379 could be associated to a second order LMR. Concerning the second order LMR it is important to observe the narrow resonance obtained for $\mathrm{SRI}=1.4189$ with a full width at half maximum of $30 \mathrm{~nm}$.

The response of this device for different SRI can be also represented as a function of the resonance wavelength (see Fig. 4). Resonance wavelength has been obtained using an algorithm on Matlab which approximated the spectral curve in a region around the peak to a parabola and determined its central axis. The refractometric response in Fig. 4 shows the calibration curve of the device, plotting the central position of the LMR as a function of the SRI value. This curve presents an average sensitivity of $7,932 \mathrm{~nm} / \mathrm{RIU}$ in a SRI range between 1.379 and 1.419. As it is typical in LMRbased refractometers, the sensitivity is increased at high SRI values. In this case the maximum sensitivity achieved was $10,336 \mathrm{~nm} / \mathrm{RIU}$ in the range 1.4079-1.4189 RIU. Concerning FWHM, the minimum value obtained with this device is 30 nm. Previous values of sensitivity and FWHM permit to obtain a commonly used performance parameter for refractometers known as figure of merit (FOM). FOM is obtained as the ratio sensitivity/FWHM and in this case is $344.5 \mathrm{RIU}^{-1}$.

TABLE I. PERFORMANCE COMPARISON

\begin{tabular}{|l|c|c|c|c|}
\hline Material & $\begin{array}{c}\text { Wavelength } \\
\text { range }(\mathbf{n m})\end{array}$ & SRI range & $\begin{array}{c}\text { Sensitivity } \\
(\boldsymbol{n m} / \boldsymbol{R I U})\end{array}$ & Ref. \\
\hline $\mathrm{CuO}\left(2^{\text {nd }} \mathrm{LMR}\right)$ & $1200-1500$ & $1.3793-1.4189$ & 7,932 & $\mathrm{~N} / \mathrm{A}$ \\
\hline $\mathrm{IGZO}$ & $1170-1550$ & $1.3950-1.4233$ & 12,929 & {$[9]$} \\
\hline $\mathrm{SnO}_{2}$ & $1380-1480$ & $1.321-1.326$ & 14,501 & {$[10]$} \\
\hline $\mathrm{ITO}$ & $1350-1650$ & $1.365-1.380$ & 8,742 & {$[11]$} \\
\hline
\end{tabular}

Performance parameters of LMR-based refractometers obtained by means of $\mathrm{CuO}$ thin-films are not better than that achieved with other coating materials such as IGZO (indiumgallium-zinc oxide) [9], $\mathrm{SnO}_{2}$ [10] or ITO (indium tin oxide) [11] as it is shown in Table 1. However, it should be noted that in this case the resonance characterized as refractometer is a second order LMR, which presents a lower sensitivity than a first order resonance (the one observed in air). Other works that compared the performance of LMRs of different order showed that the sensitivity of a first order LMR was between 3 and 7 times higher than the one of the second order LMR [7], [12]. Following conservative criteria, we could extrapolate a sensitivity of above $23,000 \mathrm{~nm} / \mathrm{RIU}$, which would demonstrate that copper oxide thin-films would be capable to provide sensitivities in the range of those achieved with tin oxide, which have demonstrated the highest sensitivity of LMR-based refractometers up to date [10], [13].

\section{CONCLUSIONS}

This research has demonstrated the possibility to use copper oxide thin-films for the development of LMR-based refractometers on a D-shaped fiber setup. The combination of a side-polished fiber and polarized light allowed to observe narrow resonances (with a FWHM of $30 \mathrm{~nm}$ ) with an average sensitivity of $7,932 \mathrm{~nm} / \mathrm{RIU}$ in the SRI range of 1.379-1.419. This LMR achieved a maximum sensitivity of $10,336 \mathrm{~nm} / \mathrm{RIU}$ and a FOM of $334.5 \mathrm{RIU}^{-1}$ in the highest SRI range. Previous figures could be further improved considering that the LMR analyzed here is a second order resonance and a first order LMR would typically achieve a performance at least 3 times higher. Therefore, it is expected that, with a proper $\mathrm{CuO}$ thin-film thickness adjustment and fabrication process monitoring, the use $\mathrm{CuO}$ could open a new path for the development of extremely sensitive LMRbased refractometers suitable to be used in the most demanding applications.

\section{ACKNOWLEDGMENT}

The authors would like to acknowledge the support from the Spanish Agencia Estatal de Investigación (AEI) (PID2019-106231RB-I00) and the Public University of Navarra (PJUPNA26). A. O. C. would also like to thank the Spanish Ministry of Universities (FPU15/05663). 


\section{REFERENCES}

[1] Q. Wang and W. M. Zhao, "A comprehensive review of lossy mode resonance-based fiber optic sensors," Optics and Lasers in Engineering, vol. 100. Elsevier, pp. 47-60, 01-Jan-2018.

[2] A. Ozcariz, C. Ruiz-Zamarreño, and F. J. Arregui, “A Comprehensive Review: Materials for the Fabrication of Optical Fiber Refractometers Based on Lossy Mode Resonance," Sensors, vol. 20, no. 7, p. 1972 , Apr. 2020.

[3] I. Del Villar et al., Optical sensors based on lossy-mode resonances, vol. 240. Elsevier B.V., 2017, pp. 174-185.

[4] F. Chiavaioli et al., "Femtomolar Detection by Nanocoated Fiber Label-Free Biosensors," ACS Sensors, vol. 3, no. 5, pp. 936-943, May 2018

[5] P. Zubiate, C. R. Zamarreño, P. Sánchez, I. R. Matias, and F. J. Arregui, "High sensitive and selective C-reactive protein detection by means of lossy mode resonance based optical fiber devices," Biosensors and Bioelectronics, vol. 93, pp. 176-181, Jul. 2017.

[6] P. Sanchez, C. R. Zamarreno, F. J. Arregui, and I. R. Matias, "LMRBased Optical Fiber Refractometers for Oil Degradation Sensing Applications in Synthetic Lubricant Oils," Journal of Lightwave Technology, vol. 34, no. 19, pp. 4537-4542, Oct. 2016.

[7] I. Del Villar et al., "Design rules for lossy mode resonance based sensors," Applied Optics, vol. 51, no. 19, p. 4298, Jul. 2012.

[8] I. Del Villar, P. Zubiate, C. R. Zamarreño, F. J. Arregui, and I. R. Matias, "Optimization in nanocoated D-shaped optical fiber sensors," Optics Express, vol. 25, no. 10, p. 10743, May 2017.

[9] A. Ozcariz, M. Dominik, M. Smietana, C. R. Zamarreño, I. Del Villar, and F. J. Arregui, "Lossy mode resonance optical sensors based on indium-gallium-zinc oxide thin film," Sensors and Actuators A: Physical, vol. 290, pp. 20-27, May 2019.

[10] F. J. Arregui, I. Del Villar, C. R. Zamarreño, P. Zubiate, and I. R. Matias, "Giant sensitivity of optical fiber sensors by means of lossy mode resonance," Sensors and Actuators B: Chemical, vol. 232, pp. 660-665, Sep. 2016.

[11] P. Zubiate et al., "High sensitive refractometers based on lossy mode resonances (LMRs) supported by ITO coated D-shaped optical fibers," Optics Express, vol. 23, no. 6, Mar. 2015.

[12] O. Fuentes, I. Del Villar, J. M. Corres, and I. R. Matias, "Lossy mode resonance sensors based on lateral light incidence in nanocoated planar waveguides," Scientific Reports, vol. 9, no. 1, p. 8882, Dec. 2019.

[13] A. Ozcariz, C. R. Zamarreño, P. Zubiate, and F. J. Arregui, "Is there a frontier in sensitivity with Lossy mode resonance (LMR) based refractometers?," Scientific Reports, vol. 7, no. 1, p. 10280, Dec. 2017. 